RESEARCH

\title{
CAN PERCUTANEOUS NEPHROLITHOTOMY BE SAFELY PERFORMED ON OCTOGENARIANS?
}

Turkish Journal of Geriatrics

DOI: 10.31086/tjgeri.2021.218

2021; 24(2): 220-226

- ilker AKARKEN ${ }^{1}$

- Hüseyin TARHAN ${ }^{1}$

- Fatih KARAÖZ

- Hasan DELIKTAŞ ${ }^{1}$

- Hayrettin ŞAHIN ${ }^{1}$

CORRESPONDANCE

${ }^{1}$ Ilker AKARKEN

Mugla Sitki Kocman University, Urology, Mugla, Turkey

\section{Phone: +905068266788}

e-mail: ilkerakarken@gmail.com

Received: Feb 17, 2020

Accepted: Apr 16, 2021

${ }^{1}$ Mugla Sitki Kocman University, Urology, Mugla, Turkey

\section{Abstract}

Introduction: This study aimed to analyze the efficacy and safety of the percutaneous nephrolithotomy procedure in octogenarians and compare them with the outcomes in a young patient group.

Materials and Methods: Patients older than 80 and younger than 90 were included in Group 1, and patients younger than 65 were assigned to Group 2. Group 1 included 45 patients, and Group 2 consisted of 90 patients recruited by one-to-two case-control matching based on gender, stone area, and the number of staghorn stones.

Results: The mean patient age was $81.89 \pm 1.70$ and $38.7 \pm 12.5$ years in Groups 1 and 2, respectively. The patients in Group 1 had a significantly higher rate of systemic hypertension. Pre-operative and post-operative serum creatinine levels were higher in Group 1 than in Group 2. The groups did not differ in terms of the preoperative serum hemoglobin level. However, the postoperative serum hemoglobin level was significantly lower in Group 1. There was no difference between the two groups in terms of the rate of blood transfusions. The duration of hospital stay was significantly longer in Group 1. However, the two groups were not different in terms of minor and major complications. The stone-free rates were calculated as $73.3 \%$ and $77.8 \%$ in Groups 1 and 2, respectively. There was no difference between the groups in terms of stone-free rates $(p=0.567)$.

Conclusion: The percutaneous nephrolithotomy procedure is an effective and safe method for treating renal stone disease in octogenarians.

Keywords: Aged, 80 and over; Nephrolithotomy, Percutaneous; Nephrolithiasis 


\section{INTRODUCTION}

Percutaneous nephrolithotomy (PCNL) has a considerable role in the surgical treatment of renal stone disease (1). PCNL has been reported to be effective and safe in treating all renal stone patients, including those in their 70s (2). In accordance with the general population's increased life expectancy over the past decades, the incidence of urinary tract stone disease has also increased in the geriatric patient population. Therefore, the rates of PCNL and other surgical interventions performed for renal stone disease in this patient population have also increased. However, cardiovascular comorbidities and the associated high American Society of Anesthesiology scores of these patients are known to complicate both the preoperative and perioperative management of these patients $(3,4)$.

Despite these reservations, the literature on the efficacy and safety of PCNL in octogenarians is scarce $(5,6)$. In this study, we aimed to compare the outcomes and complication rates of PCNL in octogenarians with those in a relatively young control group.

\section{MATERIALS AND METHODS}

The ethical review committee of our university approved this study, with approval number 200197. Data of the 450 patients who underwent PCNL at our department between January 2014 and January 2020 were retrospectively reviewed. Among these patients, those who were 80 or older constituted Group 1. The patients in Group 1 were reviewed in terms of gender, stone area, and the number of staghorn stones. These data were used to form the control group by one-to-two case-control matching among patients aged between 18 and 70 . Thus, the groups were equated in terms of the mean stone area, the number of staghorn stones, and gender distribution.

Patients younger than 18, pregnant patients, patients with bleeding diathesis, and those with con- genital renal anomalies were not included.

The PCNL procedure was performed for renal stones larger than $20 \mathrm{~mm}$. All patients underwent PCNL, provided that their urine culture was sterile. Three experienced endourologists performed all PCNL procedures. A 6F ureteral catheter was inserted into the ipsilateral ureter through rigid cystoscopy, and the renal calyceal system was punctured in a prone position by an 18G diamond-tipped needle under fluoroscopy guidance. The percutaneous access tract was dilated by Amplatz (Cook Medical LLC, Bloomington IN, US) dilators, with diameters ranging from $8 \mathrm{~F}$ to $30 \mathrm{~F}$. The renal stones were fragmented by a pneumatic lithotripter, and the stone fragments were removed by a stone grasper. A percutaneous nephrostomy tube was inserted in all PCNL cases. The ipsilateral urinary tract was scanned using flexible nephroscopy, antegrade nephrostogram, and fluoroscopy to detect residual stones immediately before the completion of the procedure. Non-contrast abdominopelvic tomography was performed if there was suspicion of clinically significant residual stone fragments postoperatively. A repeat PCNL procedure or ureteroscopy was performed for the treatment of these residual stones. In cases without clinically significant residual fragments, the nephrostomy tube and the ureter catheter were simultaneously removed after confirming the tract's patency through an antegrade nephrostogram. All patients underwent renal ultrasound or non-contrast abdominopelvic tomography at the $6^{\text {th }}$ and $12^{\text {th }}$ weeks of follow-up after discharge.

The demographic parameters and data on comorbidities, preoperative and postoperative complete blood count and blood biochemistry results, duration of hospital stay, blood transfusion, and stone-free rates and stone characteristics of the study patients were retrieved from patient folders. Moreover, the complications encountered within the postoperative 30 days were reviewed and graded as per the modified Clavien-Dindo classification 
system. The patients who did not have any clinically significant residual stone fragments during the postoperative sixth-week follow-up visit were considered "stone-free."

All statistical analyses were performed using the Statistical Package for Social Sciences (SPSS) 22.0 software (SPSS Inc., Chicago, IL, USA). The student's t-test was used to compare the continuous variables with a normal distribution, and the chi-square test was utilized to compare the categorical variables. The differences were considered significant when the $\mathrm{p}$-value was less than 0.05 .

\section{RESULTS}

The mean patient age was $81.89 \pm 1.70$ and $38.7 \pm$ 12.5 in Groups 1 and 2, respectively. Group 1 consisted of 45 patients aged 80 and 87, and Group 2 included 90 patients aged between 21 and 65 . No significant difference was found between the groups in terms of gender distribution ( $p=0.624$ ) (Table 1). A comparison of the groups in comorbidity status revealed that the rate of systemic hypertension (HT) was significantly higher in Group 1 ( $p$ $=0.038$ ). No difference was observed between the two groups in terms of the rate of other systemic

Table 1. Demographics and comparison of preop values between groups.

\begin{tabular}{|c|c|c|c|}
\hline & $\begin{array}{l}\text { Octogenarian } \\
\text { Group-1 }\end{array}$ & $\begin{array}{l}\text { Young } \\
\text { Group-2 }\end{array}$ & p-Value \\
\hline Number of Patients (n) & 45 & 90 & \\
\hline \multicolumn{4}{|l|}{ Gender } \\
\hline Female (\%) & $21(46.7)$ & $38(42.2)$ & \\
\hline Male (\%) & $24(53.3)$ & $52(57.8)$ & 0.624 \\
\hline Age $($ mean $\pm S D)$ & $81.89 \pm 1.70$ & $38.7 \pm 12.5$ & 0.001 \\
\hline \multicolumn{4}{|l|}{ Comorbidity } \\
\hline Diabetes Mellitus (\%) & $10(22.2)$ & $23(25.6)$ & 0.671 \\
\hline Hypertension (\%) & $20(44.4)$ & $24(26.7)$ & 0.038 \\
\hline Coronary Artery Disease (\%) & $11(24.4)$ & $13(14.4)$ & 0.152 \\
\hline \multicolumn{4}{|l|}{ Medications } \\
\hline Aspirin (\%) & $20(44.4)$ & $14(15.6)$ & \\
\hline Coumadin (\%) & $8(17.8)$ & $2(2.2)$ & \\
\hline Clopidogel (\%) & $13(28.9)$ & $2(2.2)$ & \\
\hline Combination (\%) & $4(8.9)$ & $0(0)$ & 0.001 \\
\hline \multicolumn{4}{|l|}{ Preop Laboratory } \\
\hline Mean Serum Creatinin $(\mathrm{mg} / \mathrm{dl} \pm \mathrm{SD})$ & $1.11 \pm 0.42$ & $0.86 \pm 0.25$ & 0.001 \\
\hline Mean Serum Hemoglobin (g/dl \pm SD) & $12.16 \pm 1.86$ & $12.63 \pm 2.12$ & 0.204 \\
\hline \multicolumn{4}{|l|}{ Stone Characteristic } \\
\hline Mean Stone Area $\left(\mathrm{mm}^{2}\right)$ & $385.17 \pm 51.47$ & $387.94 \pm 45.9$ & 0.752 \\
\hline Staghorn Stone (\%) & $12(26.7)$ & $21(23.6)$ & 0.697 \\
\hline \multicolumn{4}{|l|}{ Laterality } \\
\hline Left (\%) & $24(53.3)$ & $56(62.2)$ & \\
\hline Right (\%) & $21(46.7)$ & $34(37.8)$ & 0.322 \\
\hline
\end{tabular}


comorbid diseases. The ratio of patients on anticoagulant or antiplatelet medications was significantly higher in Group 1 than in Group 2 ( $p=0.001$ ) (Table 1).

Whereas the preoperative mean serum creatinine level was significantly higher in Group 1 than in Group 2, there was no difference between the groups in terms of the preoperative mean serum hemoglobin levels $(p=0.001$ and $p=0.204$, respectively) (Table 1).

The two groups were similar in terms of the total stone area and the number of staghorn stones due to the study cohort's case-control matched design. The postoperative mean serum creatinine level was significantly higher in Group 1 than in Group 2, whereas the postoperative mean hemoglobin level was lower in Group 1 than in Group 2 $(p=0.007$ and $p=0.029$, respectively). The number of patients that required blood transfusion was 4 (8.9\%) in Group 1 and 6 (6.7\%) in Group 2. The mean operative times of Group 1 and Group 2 were $66.12 \pm 11.47$ and $62.61 \pm 12.81$ minutes, respective- ly. No significant difference was found between the two groups in terms of blood transfusion rates and operative time ( $p=0.642$ and $p=0.124$, respectively). However, hospital stay duration was significantly longer in Group 1 than in Group 2 ( $p=0.03$ ). The comparison in complications revealed no difference between the two groups in terms of the minor (Clavien-Dindo grades 1 and 2) and major (Clavien-Dindo grades 3 and 4$)$ complication rates $(p=0.81$ ) (Table 2). Among the six patients in Group 1 who had major complications, four required retrograde double J stent (DJS) insertion because of persistent urine leakage from the nephrostomy tract, and one underwent retrograde DJS insertion because of renal colic associated with clot retention. The other patient had to undergo retrograde intrarenal stone surgery with DJS insertion because of a clinically significant residual lower pole stone. The stonefree rates were determined to be $73.3 \%$ and $77.8 \%$, respectively. A comparison of the stone-free rates revealed no statistically significant difference between the groups ( $p=0.567$ ) (Table 2).

Table 2. Comparison of postop values and complications between groups.

\begin{tabular}{|c|c|c|c|}
\hline Variable & $\begin{array}{c}\text { Octogenarian } \\
\text { Group-1 }\end{array}$ & $\begin{array}{l}\text { Young } \\
\text { Group-2 }\end{array}$ & $p$-Value \\
\hline \multicolumn{4}{|l|}{ Postop Laboratory } \\
\hline Mean Serum Creatinin $(\mathrm{mg} / \mathrm{dl} \pm \mathrm{SD})$ & $1.048 \pm 0.45$ & $0.88 \pm 0.23$ & 0.007 \\
\hline Mean Serum Hemoglobin (g/dl $\pm S D)$ & $11.2 \pm 1.59$ & $11.9 \pm 1.99$ & 0.029 \\
\hline Transfusion (\%) & $4(8.9)$ & $6(6.7)$ & 0.642 \\
\hline Operative Time (mean, minutes) & $66.12 \pm 11.47$ & $62.61 \pm 12.81$ & 0.124 \\
\hline Length of Stay (mean, day) & $2.91 \pm 1.8$ & $2.32 \pm 1.2$ & 0.03 \\
\hline \multicolumn{4}{|l|}{ Complication Rate } \\
\hline Clavien 1-2 (\%) & $11(24.4)$ & $25(27.8)$ & \\
\hline Clavien 3-4 (\%) & $6(13.4)$ & $13(13.3)$ & \\
\hline Clavien 5 (\%) & 0 & 0 & 0.081 \\
\hline Stone Free Rate (\%) & $33(73.3)$ & 70 (77.8) & 0.567 \\
\hline
\end{tabular}




\section{DISCUSSION}

The incidence of nephrolithiasis is higher in octogenarians than in the general population (7). This relatively high incidence paves the way for increased urinary tract infection and renal dysfunction rates (8) $p=0.46$. Although some of these cases benefit from conservative treatments, some mandate surgical interventions for definitive treatment. During the decision-making process, it should be considered that these patients may have impaired functional reserve with increased sensitization to complications, such as bleeding and urosepsis (9).

The potential complications of PCNL are renal hemorrhage requiring intervention (0.6\%-1.4\%), urosepsis $(0.9 \%-4.7 \%)$, pulmonary complications $(3.1 \%-23 \%)$, adjacent organ injury $(0.2 \%)$, and the requirement of a second intervention that brings additional anesthesia-related risks (10). The risk of these complications is higher in octogenarians because of the higher age of these patients and their increased rate of comorbid conditions (11). In studies investigating the data of PCNL patients older than 60 , the rate of comorbidities, such as HT, diabetes mellitus (DM), or chronic obstructive pulmonary disorder, was 66\%-88\% (12,13). In a study conducted by the Endourological Society PCNL study group that compared the outcomes of patients older than 70 with those of patients younger than 70 (i.e., 18-70 years), the rates of DM, cardiovascular diseases, and anticoagulant and prednisone use were significantly higher in the former group than in the latter (2). Thus, Morganstern et al., who compared octogenarians with young patients undergoing PCNL procedures, observed that comorbid conditions were significantly more prevalent in octogenarians (5). Consistent with our study, these authors also reported that the rate of systemic HT was significantly higher in octogenarians. In accordance with the studies that determined higher rates of antiplatelet or anticoagulant medication use, all the patients in Group 1 (i.e., octogenarians) of our study were on anticoagulant medications $(5,14)$.
The prevalence of end-stage renal disease (ESRD) was calculated as $31 \%$ in octogenarians (15). This level is significantly higher than the rate of ESRD in the general patient population (i.e., 14\%). In our study, we found a significant difference between the two groups in terms of both preoperative and postoperative serum creatinine levels. In another study, the researchers found similar results and reported that the mean serum creatinine levels were higher in octogenarians than in young patients, both preoperatively and postoperatively $(2,6,14)$.

Some studies showed no statistically significant difference between old and young PCNL patients in terms of the postoperative decrease in hemoglobin levels and blood transfusion rates $(4,5)$. Conversely, octogenarians had lower preoperative and postoperative hemoglobin levels and a higher risk of requiring blood transfusions than younger patients (6). These findings are consistent with ours.

The rate of blood transfusion increases with aging in the PCNL patient population (6). In our study, the higher blood transfusion ratio in octogenarians can be due to both older age and frequent use of anticoagulant medications.

Morganstern et al. reported that hospital stay duration was longer for octogenarians than for young patients, but the difference was not statistically significant (5). Conversely, some studies found a significantly longer mean post-PCNL hospital stay in octogenarians than in young patients (6).

Studies evaluating the success of PCNL in patients older than 65 years reported complication rates of $13 \%$ or $14 \%(4,10,16)$. Nevertheless, studies conducted on octogenarians reported complication rates of $25 \%-36 \%(5,6)$. In accordance with these reports, the complication rate was calculated as $37 \%$ in our series. Our analysis of the classification of the complications as per the modified Clavien-Dindo classification system revealed that the complication rate of octogenarians was similar to that of the young patient group. This finding is also in accordance with previous reports (5). 
Conversely, some studies reported different results (6). Abedali et al. found that Clavien-Dindo grade 2 and grade 3 complications were higher in octogenarians than in younger patients (6). These differences need to be explained by more extensive clinical trials.

Studies comparing patients older than 65 with young patients in terms of PCNL success reported stone-free rates of $70 \%-72 \%$ in both patient groups $(2,4)$. Some trials, including octogenarians undergoing $\mathrm{PCNL}$, reported stone-free rates of $72 \%-78 \%$, and the comparison of stone-free rates between octogenarians and young patients did not reveal any significant difference $(5,6)$ In our study, the octogenarians' stone-free rate was similar to that in the literature but lower than that in our young patients. However, the difference was not statistically significant.

This study has some limitations that need to be considered when evaluating its findings. First, it is a retrospective study, as a result the surgeries were not performed by the same surgeon. Second, data on the stone analysis results and the Charlson comorbidity index scores of the patients were not available. In addition, the sample size was relatively

\section{REFERENCES}

1. Ghani KR, Andonian S, Bultitude M, et al. Percutaneous Nephrolithotomy: Update, Trends, and Future Directions. Eur Urol. 2016; 70:382-96. (PMID: 26876328)

2. Okeke Z, Smith AD, Labate G, et al. Prospective comparison of outcomes of percutaneous nephrolithotomy in elderly patients versus younger patients. J Endourol. 2012; 26:996-1001. (PMID: 22494028)

3. Akman T, Binbay $M$, Sari $E$, et al. Factors affecting bleeding during percutaneous nephrolithotomy: Single surgeon experience. J Endourol. 2011; 25:327-33. (PMID: 21214412) small, more cases might have revealed further significant results. The fact that the study was carried out at a single center restricts its ability to reflect the results of the general practice. Nevertheless, considering that some urology centers hesitate to perform PCNL on octogenarians, we believe that our findings on the safety of this procedure in octogenarians are encouraging.

\section{CONCLUSIONS}

Despite the weaknesses of this study, we conclude that octogenarians may have cardiovascular or other types of comorbidities that can be challenging to both the urologists who will perform the PCNL procedure and the anesthesiologists. Nevertheless, PCNL can be safely performed in octogenarians with success and complication rates comparable with those of the young patient population.

\section{ACKNOWLEDGMENTS: -}

Conflicts of interest: The authors declare no conflicts of interest.

Financial support: None. The authors have no industrial links.

4. Nakamon T, Kitirattrakarn P, Lojanapiwat B. Outcomes of percutaneous nephrolithotomy: Comparison of elderly and younger patients. Int Braz J Urol. 2013; 39:692-701. (PMID: 24267112)

5. Morganstern B, Galli R, Motamedinia P, et al. Percutaneous nephrolithotomy in octogenarians and beyond: How old is too old? Asian J Urol. 2015; 2:20813. (PMID: 29264147)

6. Abedali ZA, Large T, Heiman JM, et al. Percutaneous Nephrolithotomy in the 80 Years of Age and Older Population. Urology. 2019; 134:62-65. (PMID: 31536740) 
7. Curhan GC. Epidemiology of Stone Disease. Urol Clin North Am. 2007; 34:287-93. (PMID:17678980)

8. Stoller ML, Bolton D, St. Lezin M, Lawrence M. Percutaneous nephrolithotomy in the elderly. Urology. 1994; 44:651-54. (PMID: 7974938)

9. Girgin R, Topaktaş R, Altın S, Aydın C, Akkoç A, Akduman B. The Effect of Charlson's Comorbidity Index on Clavien-Dindo Classification of Surgical Complications in Percutaneous Nephrolitotomy. J Urol Surg. 2016; 3:84-89. (DOI: 10.4274/jus.2016.890)

10. Skolarikos A, De La Rosette J. Prevention and treatment of complications following percutaneous nephrolithotomy. Curr Opin Urol. 2008; 18:229-34. (PMID: 18303550)

11. Seitz C, Desai M, Häcker A, et al. Incidence, prevention, and management of complications following percutaneous nephrolitholapaxy. Eur Urol. 2012; 61:146-58. (PMID: 21978422)
12. Buldu I, Tepeler A, Karatag $T$, et al. Does aging affect the outcome of percutaneous nephrolithotomy? Urolithiasis. 2015; 43:183-87. (PMID: 25395249)

13. Şahin A, Atsü N, Erdem E, et al. Percutaneous nephrolithotomy in patients aged 60 years or older. J Endourol. 2001; 15:489-91. (PMID: 11465327)

14. Ng CF. The effect of age on outcomes in patients undergoing treatment for renal stones. Curr Opin Urol. 2009; 19:211-14. (PMID: 19195134)

15. Shoag J, Halpern J, Goldfarb DS, Eisner BH. Risk of chronic and end stage kidney disease in patients with nephrolithiasis. J Urol. 2014; 192:1440-45. (PMID: 24929140)

16. Anagnostou T, Thompson T, Ng CF, Moussa S, Smith G, Tolley DA. Safety and outcome of percutaneous nephrolithotomy in the elderly: Retrospective comparison to a younger patient group. J Endourol. 2008; 22:2139-45. (PMID: 18811570) 